\title{
Criterios para la evaluación de los costos en la seguridad y salud en el trabajo: una revisión bibliográfica*
}

[Artículo de revisión]

Giovanni Andrés Ortiz Méndez ${ }^{* *}$

Recibido: 11 de julio de 2020

Revisado: 8 de marzo de 2021

Aceptado: 13 de marzo de 2021

Citar como:

Ortiz Méndez, G. A. (2021). Criterios para la evaluación de los costos en la seguridad y salud en el trabajo: una revisión bibliográfica. Signos, Investigación en Sistemas de Gestión, 13(2). https://doi.org/10.15332/24631140.6671

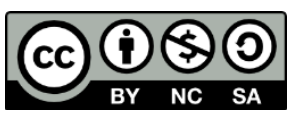

\section{Resumen}

La finalidad de este artículo es presentar los resultados de una revisión de la literatura para identificar los referentes teóricos que aporten a la construcción de una metodología de la evaluación de costos en seguridad y salud en el trabajo. La revisión se hizo a 26 publicaciones de 3 bases de datos, incluyendo los artículos encontrados a través de la búsqueda iterativa. Para el análisis de la información se elaboró una matriz de

\footnotetext{
* Artículo de revisión.

** Magíster en Calidad y Gestión integral por la Universidad Santo Tomás, especialista en Salud Ocupacional y Riesgos Laborales por la Universidad Manuela Beltrán e ingeniero ambiental por la Universidad Santo Tomás, Bogotá, Colombia. Correo: giovanniortiz@usantotomas.edu.co, giovandresor@hotmail.com; ORCID: https://orcid.org/0000-0002-5101-9831; CvLAC:

https://scienti.minciencias.gov.co/cvlac/visualizador/generarCurriculoCv.do?cod rh $=0000005037$
}

Signos, Investigación en Sistemas de Gestión

ISSN: 2145-1389 | e-ISSN: 2463-1140 | DOI: https://doi.org/10.15332/24631140

Vol. 13 N.0 2 | julio-diciembre de 2021 
resumen analítico especializado. Este estudio permitió identificar que actualmente se están realizando en el mundo estudios sobre seguridad y salud en el trabajo que incluyen evaluaciones de costos. Además, se pudo determinar los referentes teóricos que aportaran a la construcción de una metodología de evaluación de costos en seguridad y salud en el trabajo: los costos directos, los costos indirectos y los indicadores (evaluaciones económicas); y los análisis que se han realizado a los diferentes criterios que establece cada uno.

Palabras clave: evaluación de costos, seguridad y salud en el trabajo, sistema de gestión de seguridad y salud en el trabajo, prevención.

\section{Criteria for evaluating occupational safety and health costs: a literature review}

\section{Abstract}

This article's purpose is to present the results of a literature review to identify the theoretical references that contribute to develop a methodology for the evaluation of occupational safety and health costs. Twenty-six (26) publications from 3 databases were reviewed, including articles found through an iterative search. For analyzing the information, a specialized analytical summary matrix was developed. This study made it possible to identify that studies on occupational safety and health including cost evaluations are currently being carried out in the world. Moreover, it was possible to determine the theoretical references that would contribute to developing a cost evaluation methodology in occupational safety and health: direct costs, indirect costs and indicators (economic evaluations); and the analyzes that have been carried out on the different criteria established by each one.

Keywords: cost evaluation, occupational health and safety, occupational health and safety management system, prevention. 


\section{Critérios para a avaliação dos custos de segurança e saúde ocupacional: uma revisão bibliográfica}

\section{Resumo}

Este artigo visa apresentar os resultados de uma revisão bibliográfica para identificar as referências teóricas que contribuem para a criação de uma metodologia de avaliação de custos em segurança e saúde ocupacional. A revisão foi feita com 26 publicações de 3 bancos de dados, incluindo os artigos encontrados através da busca iterativa. Para analisar as informações, foi desenvolvida uma matriz de resumo analítico especializado. Esse estudo ajudou a identificar que atualmente em todo o mundo estao sendo desenvolvidos estudos sobre segurança e saúde ocupacional que incluem avaliações de custos. Além disso, foi possível determinar as referências teóricas que contribuem para a criação de uma metodologia de avaliação dos custos em segurança e saúde ocupacional: custos diretos, custos indiretos e indicadores (avaliações econômicas); e as análises que foram feitas para os diferentes critérios estabelecidos por cada um.

Palavras-chave: avaliação de custos, segurança e saúde ocupacional, sistema de gestão de segurança e saúde ocupacional, prevenção.

\section{Introducción}

La normativa colombiana sobre riesgos laborales o seguridad y salud en el trabajo (SST) es cada día más exigente para las empresas. Desde la creación de la Ley 1562 de 2012 (la cual modifica el sistema de riesgos laborales), las empresas han tenido que prestarle una mayor importancia a los peligros a los que están expuestos sus trabajadores y se han visto obligadas a implementar un sistema de gestión de seguridad y salud en el trabajo (SG-SST). 
El Decreto 1072 de 2015 (Decreto Único Reglamentario del Sector Trabajo, DUR) es la norma que establece las directrices de obligatorio cumplimiento que deben seguir las empresas para diseñar e implementar sus SG-SST. Este decreto tiene dentro de las obligaciones de los empleadores la asignación de recursos financieros para garantizar el funcionamiento del SG-SST, así mismo, pide que las empresas hagan una revisión donde analicen la suficiencia de los recursos asignados. Es importante anotar que, en Colombia, la implementación del SG-SST en cada organización debe ser liderada e implementada por el empleador y debe ser coherente con los lineamientos dictados por el Gobierno nacional para el cuidado de los trabajadores (Álvarez Torres y Riaño Casallas, 2018).

En el año 2019, la Resolución 0312 de 2019 ordenó que las empresas asignen recursos económicos para desarrollar acciones de promoción de la salud y prevención de los riesgos laborales (PyP). Para la asignación de estos recursos económicos, las empresas deben llevar un presupuesto de sus SG-SST y no existe una norma que obligue a aplicar una metodología definida para hacer este presupuesto o para realizar los diferentes análisis de costos en SST.

En la literatura existen diversas metodologías y una gran cantidad de trabajos sobre las evaluaciones económicas en tecnologías de ciencias de la salud (Drummond et ál., 2015); sin embargo, son menos los que se han concentrado en analizar la relación entre el trabajo y la salud, los costos asociados a los accidentes de trabajo y las enfermedades laborales (ATEL), incluyendo las implicaciones para las organizaciones. Algunas investigaciones se han centrado en sectores de alta accidentalidad como el sector de la construcción; por ejemplo, el estudio de Arboleda Giraldo (2019) relaciona las dificultades en la gestión de la SST al no contar con 
personal capacitado y no recibir de las empresas la información necesaria a los encargados de SST para implementar adecuadamente sus SG-SST. Revisiones realizadas previamente sobre las evaluaciones económicas en SST demuestran que el tema está comenzando a explorarse y que los avances investigativos que se tienen son provenientes de otros países del mundo que no hablan español (M. Riaño Casallas y Palencia Sánchez, 2016). Por esto, se hizo una revisión de literatura en diferentes bases de datos para recopilar la información más relevante (Guirao Goris et ál., 2008) e identificar los referentes teóricos que le permitan al lector entender cómo se han evaluado los costos en SST y que sea una base teórica para los tomadores de decisiones sobre SST de las organizaciones.

En países como Perú y España hay avances de propuestas metodológicas que presentan una guía para el cálculo de los costos de los accidentes de trabajo y las enfermedades laborales (INSHT, 1999, 2001, 2009; Junta de Castilla y León, 2005; Ministerio de trabajo y promoción de empleo, 2017, 2018); también se encuentra una guía de la OMS que cumple el objetivo de orientar a los tomadores de decisiones en el proceso del cálculo de los costos (Mossink, 2004). En el caso de Italia, se están proponiendo metodologías que miden la implementación efectiva de los SG-SST aplicables a empresas de la Unión Europea (Bianchini et ál., 2017). Es importante destacar que existen también metodologías de evaluación de costos en otros sistemas de gestión, como el de calidad (Torres Navarro y Callegari Malta, 2016).

\section{Metodología}

La revisión de la literatura fue realizada a 26 publicaciones depuradas del total de 89 publicaciones encontradas. La búsqueda inicial se realizó en tres bases de datos: ScienceDirect, JSTOR y Taylor \& Francis. La búsqueda se realizó con la siguiente fórmula: “cost evaluation” AND “occupational 
safety and health". De esta búsqueda se incluyeron 2 publicaciones para estudio.

También se vincularon 24 documentos identificados por medio de la búsqueda iterativa, los cuales corresponden a artículos de investigación, artículos de revisión y tesis que se relacionan directamente con las variables de la investigación.

Estos documentos fueron revisados utilizando la técnica de matriz resumen analítico especializado (RAE), la cual consiste en la condensación de información contenida en documentos y estudios de una manera que facilite al lector o usuario la aprehensión y análisis del documento en cuestión (Universidad Pedagógica Nacional, 2017); en este caso se utilizó para detectar los criterios que abordan las publicaciones sobre las evaluaciones de costos en la gestión de la SST. La figura 1 explica el procedimiento utilizado para la depuración de la información de esta revisión. 
Figura 1. Procedimiento de depuración

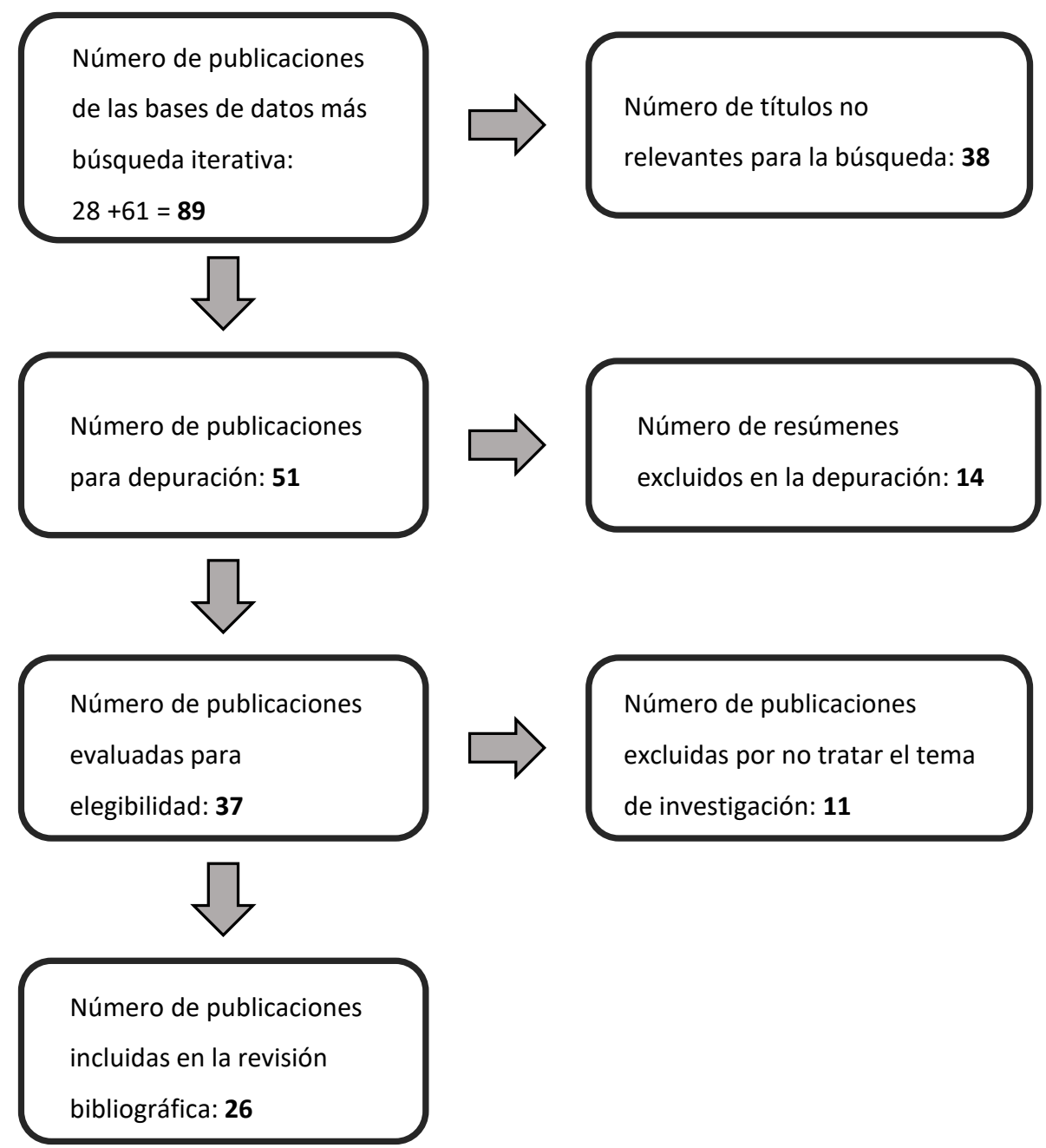

Fuente: elaboración propia.

\section{Resultados y discusión}

Luego de analizar las publicaciones, se encontró en las bases de datos que son 3 las variables que se tienen en cuenta para el análisis de evaluaciones de costos en SST. Estas variables a su vez contienen los diferentes criterios que se utilizan en SST y que pueden utilizar las organizaciones para evaluar los costos de SG-SST. En la tabla 1 se resumen las variables encontradas y los diferentes criterios de evaluación de costos que se 
plantean en las publicaciones incluidas en la revisión bibliográfica, así como el número de publicaciones incluidas y que están asociadas al criterio con los respectivos estudios que lo soportan.

Tabla 1. Variables y criterios que sirven para definir criterios de evaluación de costos en SST

\begin{tabular}{|c|c|c|c|}
\hline Variables & Criterio & $\begin{array}{c}\text { Número de } \\
\text { publicaciones } \\
\text { asociadas al criterio }\end{array}$ & Estudios de soporte \\
\hline \multirow{3}{*}{ 1. Costos directos } & $\begin{array}{c}\text { Costo de } \\
\text { indemnización de la } \\
\text { víctima del accidente. }\end{array}$ & 1 & Cuervo Vahos (2019). \\
\hline & $\begin{array}{l}\text { Costo de } \\
\text { hospitalización y } \\
\text { servicio de } \\
\text { ambulancia. }\end{array}$ & 4 & $\begin{array}{c}\text { Ramos et ál. (2016); M. } \\
\text { Riaño Casallas y Palencia } \\
\text { Sánchez (2015); Cuervo } \\
\text { Vahos (2019); Bolaño } \\
\text { Fandiño (2019). }\end{array}$ \\
\hline & $\begin{array}{l}\text { Gastos médicos } \\
\text { (incluidos los costos de } \\
\text { rehabilitación). }\end{array}$ & 5 & $\begin{array}{l}\text { Ramos et ál. (2016); Leigh } \\
\text { (2011); Franche et ál. } \\
\text { (2005); M. Riaño Casallas y } \\
\text { Palencia Sánchez (2015); } \\
\text { Cuervo Vahos (2019). }\end{array}$ \\
\hline \multirow{4}{*}{$\begin{array}{l}\text { 2. Costos } \\
\text { indirectos }\end{array}$} & $\begin{array}{c}\text { Costo legal y } \\
\text { administrativo. }\end{array}$ & 5 & $\begin{array}{c}\text { Jallon et ál. (2011); Steel } \\
\text { et ál. (2018a); Cuervo Vahos } \\
\text { (2019); Bolaño Fandiño } \\
\text { (2019); Vanegas Serna } \\
\text { (2014). }\end{array}$ \\
\hline & $\begin{array}{l}\text { Costos de } \\
\text { productividad. }\end{array}$ & 10 & $\begin{array}{l}\text { Jallon et ál. (2011); Steel } \\
\text { et ál. (2018a); Acevedo } \\
\text { González y Yánez Contreras } \\
\text { (2016); Steel et ál. (2018b); } \\
\text { Leigh (2011); Uegaki et ál. } \\
\text { (2010); M. Riaño Casallas y } \\
\text { Palencia Sánchez (2015); } \\
\text { Cuervo Vahos (2019); } \\
\text { Bolaño Fandiño (2019); } \\
\text { Vanegas Serna (2014). }\end{array}$ \\
\hline & Costos de reemplazo. & 5 & $\begin{array}{c}\text { Jallon et ál. (2011); Franche } \\
\text { et ál. (2005); M. Riaño } \\
\text { Casallas y Palencia Sánchez } \\
\text { (2015); Cuervo Vahos } \\
\text { (2019); Bolaño Fandiño } \\
\text { (2019). }\end{array}$ \\
\hline & $\begin{array}{l}\text { Costos de la } \\
\text { investigación. }\end{array}$ & 4 & $\begin{array}{l}\text { Jallon et ál. (2011); Cuervo } \\
\text { Vahos (2019); Bolaño }\end{array}$ \\
\hline
\end{tabular}

Signos, Investigación en Sistemas de Gestión

ISSN: 2145-1389 | e-ISSN: 2463-1140 | DOI: https://doi.org/10.15332/24631140

Vol. 13 N.० 2 | julio-diciembre de 2021 


\begin{tabular}{|c|c|c|c|}
\hline Variables & Criterio & $\begin{array}{c}\text { Número de } \\
\text { publicaciones } \\
\text { asociadas al criterio }\end{array}$ & Estudios de soporte \\
\hline & & & $\begin{array}{c}\text { Fandiño (2019); Vanegas } \\
\text { Serna (2014). }\end{array}$ \\
\hline \multirow{4}{*}{ 3. Indicadores } & $\begin{array}{l}\text { Análisis de costo- } \\
\text { efectividad (ACE). }\end{array}$ & 8 & $\begin{array}{l}\text { Adeyemi et ál. (2020); M. } \\
\text { Riaño Casallas y Palencia } \\
\text { Sánchez (2016); Van } \\
\text { Dongen et ál. (2014); Lee } \\
\text { (2018); Tompa et ál. (2008); } \\
\text { Tompa et ál. (2006); Grimani } \\
\text { et ál. (2018); Zárate (2010). }\end{array}$ \\
\hline & $\begin{array}{l}\text { Análisis de costo- } \\
\text { beneficio (ACB). }\end{array}$ & 11 & $\begin{array}{l}\text { M. Riaño Casallas y Tompa } \\
\text { (2018); M. Riaño Casallas y } \\
\text { Palencia Sánchez (2016); } \\
\text { Van Dongen et ál. (2014); } \\
\text { Tompa et ál. (2009); Tompa } \\
\text { et ál. (2013); Tompa et ál. } \\
\text { (2008); Tompa et ál. (2006); } \\
\text { M. I. Riaño Casallas, (2017); } \\
\text { Zárate (2010); Vélez Ávila } \\
\text { (2018); Álvarez Enciso et ál. } \\
\text { (2019). }\end{array}$ \\
\hline & $\begin{array}{c}\text { Análisis de costo- } \\
\text { utilidad (ACU). }\end{array}$ & 3 & $\begin{array}{c}\text { M. Riaño Casallas y Palencia } \\
\text { Sánchez (2016); Van } \\
\text { Dongen et ál. (2014); Zárate } \\
\text { (2010). }\end{array}$ \\
\hline & $\begin{array}{l}\text { Análisis de } \\
\text { minimización de } \\
\text { costos. }\end{array}$ & 2 & $\begin{array}{l}\text { Van Dongen et ál. (2014); } \\
\text { Zárate (2010). }\end{array}$ \\
\hline
\end{tabular}

Fuente: elaboración propia.

La mayoría de las publicaciones (el 62 \%) fueron realizadas entre los años 2014 y 2019, aspecto que evidenció que actualmente están en estudio las evaluaciones de costos en SST, y el $42 \%$ de estas publicaciones se encontraron en el idioma español, el restante en el idioma inglés (ver figura 2). 
Figura 2. Número de publicaciones incluidas por año

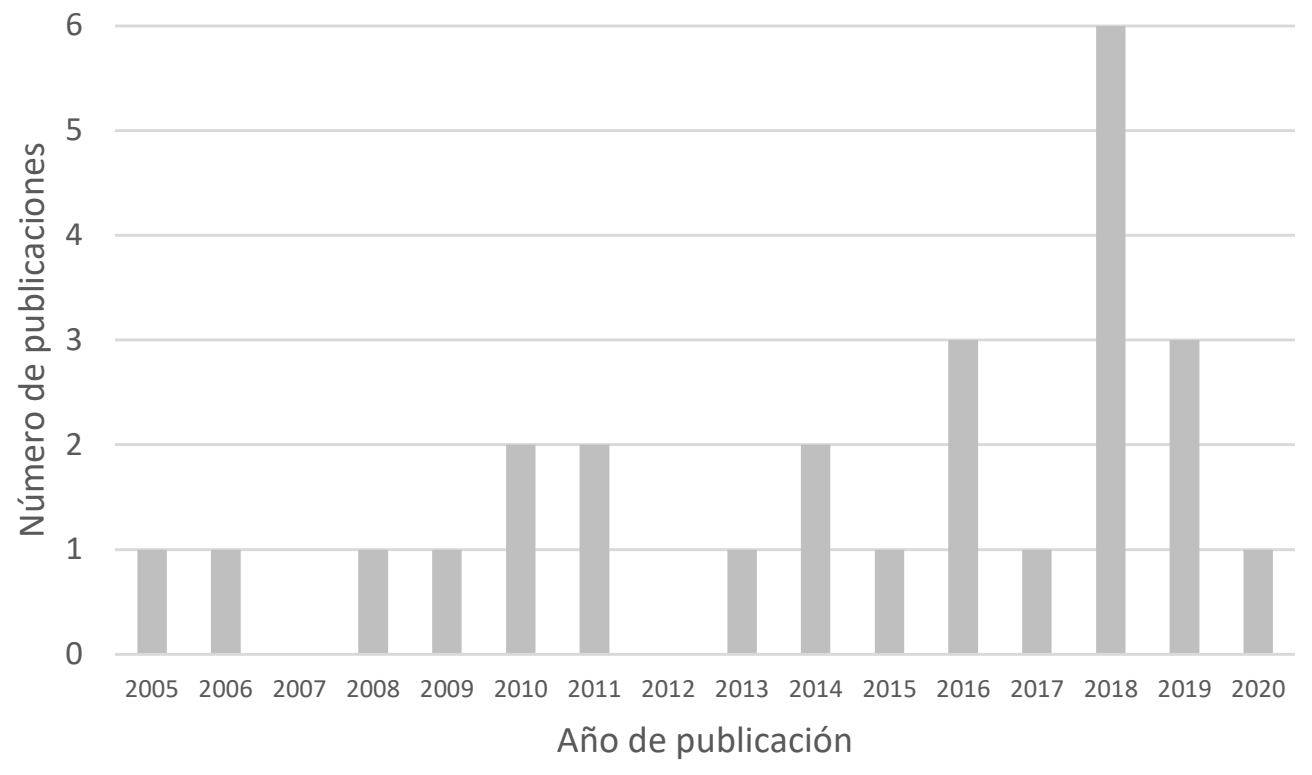

Fuente: elaboración propia.

\section{Contexto}

Es importante destacar que, en las 26 publicaciones incluidas en el estudio, se encuentran tesis de grado, estudios de aplicación de metodologías, descripción de metodologías e inclusive artículos de revisión y comparación de teorías. Dentro de las variables identificadas en la literatura, se hallaron los siguientes aspectos a destacar:

\section{Costos directos}

Según se puede identificar en la literatura, existen diversos estudios sobre los costos directos en evaluaciones económicas, esto ya que los costos son los más comunes y fáciles de identificar, pero no corresponden a estudios de la última década. De las 26 publicaciones incluidas, se encontró que 6 mencionan el tema de los costos directos. 


\section{Costo de indemnización de la víctima del accidente}

Estos costos se refieren a aquellos casos específicos en los que la empresa debe realizar un pago único a la víctima del accidente según indican las normas laborales. Solo un estudio comparativo menciona directamente estos costos, el cual buscaba integrar diferentes metodologías para revisar, analizar e interpretar los modelos de costos establecidos en el mundo, para seleccionar un modelo de estimación del costo de un accidente de trabajo en Colombia (Cuervo Vahos, 2019).

\section{Costo de hospitalización y servicio de ambulancia}

Se refieren al traslado del lesionado o enfermo a un centro hospitalario, los gastos de hospitalización, los implementos médicos, los medicamentos y el tratamiento (Cuervo Vahos, 2019). Por lo general, estos costos se pueden calcular de una manera sencilla, ya que se puede solicitar la información de los registros contables de los centros hospitalarios (Riaño Casallas y Palencia Sánchez, 2015). En algunos casos puede ser visto como una externalidad y ser incluido en los análisis de costos (Ramos et ál., 2016), mientras que en otros casos las organizaciones pueden haber realizado directamente el traslado del trabajador al centro de salud más cercano y estas deben incluir este costo en sus análisis (Bolaño Fandiño, 2019).

\section{Gastos médicos (incluidos los costos de rehabilitación)}

Estos son los costos para la sociedad en términos de pago de hospitalización, tratamientos y recuperación (combinando los costos anteriores de hospitalización con los gastos médicos) (Ramos et ál., 2016). Estos son fundamentales en los estudios donde se estima la carga económica de accidentes y enfermedades, ya que pueden presentarse casos con costos de rehabilitación bastante altos y durante períodos de largo tiempo (Leigh, 2011). Se encontró también que la duración de la incapacidad y los costos médicos asociados se reducen significativamente 
cuando se realizan intervenciones al lugar de trabajo (Franche et ál., 2005). En muchas investigaciones estos costos están incluidos (Cuervo Vahos, 2019) y se ha podido identificar que requieren altas sumas de dinero (Riaño Casallas y Palencia Sánchez, 2015).

Este tipo de costos ha sido estudiado ampliamente desde las publicaciones de Heinrich en 1931 cuando clasificó los costos de los accidentes de trabajo en directos e indirectos, siendo la clasificación más usada a nivel mundial (Heinrich, 1931); sin embargo, existen otras tendencias como la de Grimaldi y Simonds en 1996, en la cual propusieron clasificar los costos de los accidentes de trabajo en dos clases básicas: los costos asegurados y los no asegurados (Grimaldi y Simonds, 1996). Como existe una gran similitud entre los costos directos y los costos asegurados, quizás este sea el motivo por el cual hay pocos estudios sobre este tema en la actualidad, ya que analizar y calcular los costos asegurables no resultan ser una necesidad puntual o novedosa para las empresas. En el caso puntual de Colombia, la responsabilidad de estos costos se comparte con la administradora de riesgos laborales (ARL) a la cual esté afiliado el trabajador, en las cuales se denominan las prestaciones económicas y asistenciales.

\section{Costos indirectos}

En esta dimensión se encontraron más estudios. De las 22 publicaciones incluidas, se encontró que 11 mencionan el tema de los costos indirectos. Según lo planteado por Heinrich, los costos indirectos son 4 veces más que los costos directos (Heinrich, 1931).

Costo legal y administrativo

Estos corresponden a recursos financieros y humanos para trámites de registro, compilar estadísticas de accidentes, emitir informes, etc. (Jallon 
et ál., 2011). También, se consideran los honorarios de los abogados cuando existen demandas impuestas por los trabajadores, el SG-SST y las normas generales sobre riesgos laborales o seguridad laboral (Cuervo Vahos, 2019; Vanegas Serna, 2014). En una investigación reciente, se incluyen las sanciones o penalizaciones por incumplimientos laborales, ambientales y a las normas de SST que haya generado el accidente de trabajo (Bolaño Fandiño, 2019).

Independientemente de su eficacia, las amplias normas de seguridad complican la economía, ya que los programas de seguridad y salud ocupacional implican una importante gestión administrativa, inversión de carga y tiempo para los empleadores, aunque las empresas que gestionan bien pueden llegar a tener subsidios o reducciones de impuestos (Steel et ál., 2018a).

\section{Costos de productividad}

Son todos aquellos que suceden como consecuencia de una situación imprevista en la que se interrumpe el equilibrio laboral, como las paras de trabajo, horas extras, demoras en la producción, etc. (Jallon et ál., 2011); también pueden presentarse fallas de cumplimiento con los clientes (Acevedo González y Yánez Contreras, 2016). La productividad para las empresas se reducirá para el accidentado, los empleados que auxiliaron al lesionado, baja en la motivación, la falta de atención de quienes presenciaron el accidente y los daños en la propiedad que afecten la producción (Cuervo Vahos, 2019). Este costo también es definido como el costo de paro del proceso, definido como el costo incurrido por la empresa para suplir el paro del proceso (Bolaño Fandiño, 2019; Vanegas Serna, 2014).

A pesar de que la productividad baja, también se pueden observar algunos beneficios de calcular los costos de productividad al tener mayor control 
en las estadísticas de producción, presentismo y ausentismo (Steel et ál., 2018b).

Existen varios estudios que cuantifican la productividad y su relación con la SST (Acevedo González y Yánez Contreras, 2016; Cuervo Vahos, 2019; Jallon et ál., 2011; Leigh, 2011; Riaño Casallas y Palencia Sánchez, 2015; Steel et ál., 2018a; Steel et ál., 2018b; Uegaki et ál., 2010). La productividad es un elemento clave del atractivo económico de invertir en SST (Steel et ál., 2018b). Se determinó en un estudio que incluye costos directos e indirectos, que los costos de productividad son mucho más altos de lo que se piensa, ya que todos los miembros de la sociedad involucrados comparten esta carga (Leigh, 2011). También, otro estudio relaciona que los métodos para medir y valorar la productividad relacionada con la salud varían ampliamente, lo que dificulta la comparabilidad de los resultados y la toma de decisiones (Uegaki et ál., 2010).

La pérdida de productividad, cuando ocurre un ATEL, no solo afecta a las empresas, sino a la familia de la persona afectada, ya que se disminuyen los salarios y la capacidad adquisitiva en el hogar del trabajador afectado (Riaño Casallas y Palencia Sánchez, 2015).

\section{Costos de reemplazo}

Son todos aquellos que suceden como consecuencia de una situación imprevista en la que un trabajador debe ausentarse como consecuencia de un ATEL y en su reemplazo se debe mantener la productividad. Se incurre en costos en la búsqueda de personal y la gestión para transferir, seleccionar, contratar y capacitar personal (Bolaño Fandiño, 2019; Cuervo Vahos, 2019; Jallon et ál., 2011; Riaño Casallas y Palencia Sánchez, 2015). El trabajador agregado no está incluido en la evaluación de los análisis de producción o el cálculo de costos versus productividad. En este mismo 
estudio, se determinó que la evidencia en la literatura sobre el impacto de los reemplazos fue insuficiente (Franche et ál., 2005).

\section{Costos de la investigación}

Estos costos son los derivados de la investigación de las causas del accidente de trabajo y todo el tiempo utilizado por la organización para realizar la documentación administrativa pertinente y los asuntos legales que se deban cumplir (Acevedo González y Yánez Contreras, 2016; Bolaño Fandiño, 2019; Jallon et ál., 2011; Vanegas Serna, 2014). Estos también incluyen los honorarios a pagar a quienes se encarguen de la investigación, reporte y de generar medidas de prevención (Cuervo Vahos, 2019).

Este tipo de costos (los indirectos) incluyen el tiempo perdido del personal lesionado y de los involucrados, la interrupción en la producción, entre otros. Atendiendo a la premisa planteada por Heinrich, de que los costos indirectos son 4 veces superiores a los costos directos, se destaca su importancia y su estudio a fondo. Hoy en día se cuenta con información contable que, con ayuda de nuevos recursos tecnológicos, facilitan la manera de calcular estos costos, y también la forma en la que se pueden analizar en las organizaciones.

En el año 2011, un estudio reveló que en los Estados Unidos los costos indirectos para los accidentes y enfermedades fatales y no fatales ocurridas en ese país durante el año 2007 fueron casi 3 veces superiores que los costos directos (Leigh, 2011). Todo esto resalta la importancia que tiene su medición y su análisis para las evaluaciones económicas de la SST.

Se relacionan en el presente artículo algunos estudios provenientes de países de habla hispana que han generado propuestas para el cálculo de los costos de los accidentes de trabajo, entre las cuales se integran los costos directos e indirectos (Bolaño Fandiño, 2019; Carvajal Peláez y Pellicer Armiñana, 2011; Cuervo Vahos, 2019). Otro estudio realizó una 
comparación entre el método de Wallach, Grimaldi y Simonds y el de Heinrich para calcular los costos de accidentes de trabajo del sector floricultor (Díaz Rodríguez, 2019); y, finalmente, otro estudio identificó los costos directos e indirectos relacionados con ATEL basándose en las estadísticas de una empresa metalmecánica (Rincón Ramírez y Delgado Villarreal, 2018).

\section{Indicadores}

En esta dimensión se encontraron más publicaciones que en las otras dos. De las 22 publicaciones incluidas, se encontró que 13 mencionan el tema de los indicadores, el cual se refiere a evaluaciones económicas aplicadas a la gestión de la SST.

\section{Análisis de costo-efectividad (ACE)}

Estos miden los costos y algunas consecuencias, por ejemplo, la productividad y las implicaciones en la utilización de la atención médica. Son medidas en unidades monetarias, mientras que el resultado clave se mide en unidades naturales (Van Dongen et ál., 2014). En estos análisis los beneficios de los programas a evaluar no son equivalentes y son medidos en unidades naturales de morbilidad, mortalidad o calidad de vida. Las unidades utilizadas con frecuencia son las muertes evitadas, los años de vida ganados, cambios en escalas de calidad de vida relacionada con la salud, entre otros (Zárate, 2010).

Varios estudios resaltan la efectividad de las intervenciones en SST como el realizado en ergonomía para cargadores de camiones en Nigeria (Adeyemi et ál., 2020), así mismo, el análisis de casos realizado por Lee (2018); y la revisión sistemática de Tompa et ál. (2008) de las intervenciones de gestión de la discapacidad. 
Una conclusión es que en esta área hay pocos estudios y los que existen tienen una baja calidad (Riaño Casallas y Palencia Sánchez, 2016). Tompa et ál. (2006) resaltaba que estos estudios tienen deficiencias metodológicas y Grimani et ál. (2018) lo recalcan cuando afirma la necesidad de una evidencia económica de alta calidad para evaluar la relación costo-efectividad de las intervenciones de SST, especialmente a nivel organizacional, en todas las áreas de la salud de los trabajadores.

\section{Análisis de costo-beneficio (ACB)}

Los análisis costo-beneficio se han utilizado históricamente para estimar todos los costos y beneficios de un proyecto propuesto en valor monetario, y también para tener medidas como el valor presente neto o la razón costobeneficio (Riaño Casallas, 2017). Tanto los costos como las consecuencias son medidos en unidades monetarias. En administración de empresas, el $\mathrm{ACB}$ a veces se describe como el análisis de retorno de la inversión (Van Dongen et ál., 2014) y estos análisis permiten al analista realizar comparaciones directas entre distintas alternativas a través de la ganancia monetaria neta o de la razón de costo-beneficio (Zárate, 2010).

Existen varios estudios en este campo (Riaño Casallas y Palencia Sánchez, 2016): en el año 2018 Riaño Casallas y Tompa realizaron un estudio en Colombia que proporciona nueva evidencia empírica sobre la eficacia y el costo-beneficio de las inversiones en SST (Riaño Casallas y Tompa, 2018). Se encontró otro estudio que resalta la importancia de implementar metodologías y evaluar su beneficio para talleres automotrices (Vélez Ávila, 2018). También se encontraron estudios más elaborados entre los que se destacan: una evaluación económica de un proceso de ergonomía participativa en un fabricante de autopartes (Tompa et ál., 2009) y para una planta textil (Tompa et ál., 2013). En ambos se resalta que el proceso de ergonomía participativa puede ser rentable para una empresa. así 
mismo, otro estudio que destaca los beneficios financieros de las intervenciones de gestión de la discapacidad para un grupo de la industria (Tompa et ál., 2008). Por último, otro estudio estableció que el ACB conviene como herramienta para la toma de decisiones frente al SG-SST en el sector hotelero (Álvarez Enciso et ál., 2019).

\section{Análisis de costo-utilidad (ACU)}

Los costos y algunas consecuencias son medidos en términos monetarios, mientras que el resultado clave es la medición asegurada en unidades de servicios públicos. Las utilidades normalmente se expresan en términos de años de vida ajustados por calidad (AVAC) (Van Dongen et ál., 2014). Este tipo de análisis es multidimensional porque examina como beneficio una unidad común que considera tanto la calidad de vida como la cantidad o largo de vida obtenida como consecuencia de una intervención. Las unidades que se usan frecuentemente para medir los beneficios en los ACU son los años de vida ajustados por la calidad, los años de vida ajustados por la discapacidad y los años saludables equivalentes (Zárate, 2010). Existen estudios que resaltan este tipo de evaluación económica y los beneficios sociales que tienen estas (Riaño Casallas y Palencia Sánchez, 2016).

\section{Análisis de minimización de costos (AMC)}

Solo se consideran los costos en todas las alternativas, ya que se supone que las consecuencias son similares. Los análisis de minimización de costos se consideran inapropiados si existe incertidumbre con respecto a una posible diferencia en la magnitud de las consecuencias (Van Dongen et ál., 2014). Este tipo de análisis compara exclusivamente los costos de dos intervenciones alternativas bajo la premisa de que las dos proveen un nivel de beneficio equivalente, es por esto que en la práctica hay pocos 
análisis de este tipo, por la dificultad de que dos intervenciones generen los mismos beneficios (Zárate, 2010).

Estos análisis de evaluaciones económicas, aplicables a la gestión de la SST, son una fuente bastante amplia de información y son temas que actualmente se encuentran en estudio. A pesar de que la mayoría de las investigaciones no corresponden a evaluaciones aplicadas, se puede evidenciar que en los últimos años se están aplicando más este tipo de evaluaciones a programas específicos de SST en diferentes organizaciones. Este tipo de análisis permite saber si vale la pena invertir en un programa al compararlo con otro, también permite priorizar y estimar la magnitud de las consecuencias de una acción en SST. Por lo anterior, la evaluación económica es la herramienta que facilita el proceso de tomar decisiones (Riaño Casallas, 2017).

Para la Organización Mundial de la Salud (OMS) estos análisis son muy importantes, y por esto se puede contar con una guía publicada en el año 2004, la cual presenta un documento para fomentar las prácticas correctas en estos análisis de costos que tienen que ver con evaluaciones económicas de costo-efectividad, costo-beneficio, los costos de intervención (gestión de la SST) y de los ATEL (Mossink, 2004).

Finalmente, todos los avances recopilados en este artículo son una fuente para la identificación de criterios de costos en SST. Es importante saber que así como existen bastantes avances teóricos, actualmente se están aplicando estas metodologías, e inclusive discutiéndolas y analizándolas (Steel et ál., 2018a; Tompa et ál., 2010; van Dongen et ál., 2013); también hay quienes están creando nuevos modelos de estimación de estos costos (Bolaño Fandiño, 2019; Carvajal Peláez y Pellicer Armiñana, 2011; Cuervo Vahos, 2019; Vanegas Serna, 2014), lo cual supone un tema para continuar explorando e investigando. En Colombia y en Latinoamérica los estudios 
que relacionan los costos con la gestión de la SST son muy limitados y se puede contemplar esto como una gran oportunidad para realizar investigaciones profundas en el tema (Riaño Casallas y Palencia Sánchez, 2016).

\section{Conclusiones}

La mayoría de las investigaciones seleccionadas para estudio fueron realizadas en la última década, del total de publicaciones seleccionadas para el estudio, el $62 \%$ se publicaron entre 2014 y 2019, un aspecto que evidenció que a nivel mundial se están realizando estudios de la gestión de la SST y su relación con las evaluaciones de costos.

Se evidenció que el tema se ha abordado desde países que no son de habla hispana, principalmente publicados en revistas de seguridad y salud en el trabajo en el idioma inglés (el $58 \%$ ), esto permite determinar que en Latinoamérica hacen falta más estudios económicos que relacionen las evaluaciones económicas y los análisis de costos en la gestión de la SST.

Del total de 26 publicaciones, se encontraron 10 publicaciones que incluyeron aspectos metodológicos o revisiones bibliográficas y solamente 7 estudios aplicados a programas de SST. Esto repercute en que los tomadores de decisiones y las organizaciones no cuenten con una guía que les permita medir el impacto económico de sus programas de SST o los beneficios económicos de sus SG-SST.

Se pudo determinar los referentes teóricos que aportaran a la construcción de una metodología de evaluación de costos en SST: los costos directos, los costos indirectos y los indicadores (evaluaciones económicas); y los análisis que se han realizado a los diferentes criterios que establece cada uno. 
En la presente revisión de literatura se evidenció que los criterios más mencionados en la literatura son los costos de productividad y el análisis costo-beneficio. También hay países como Perú y España que cuentan con algunas guías para calcular los costos de los ATEL.

Se resalta en la bibliografía la importancia de realizar un análisis de costos en las organizaciones y los beneficios que conlleva realizar este tipo de estudios para los tomadores de decisiones de las organizaciones. Es importante poder conocer las diferentes metodologías, para así poder evaluar y determinar los beneficios de una buena gestión de la SST para la sociedad, las empresas, los trabajadores y las demás partes interesadas.

\section{Referencias}

Acevedo González, K., y Yánez Contreras, M. (2016). Costos de los accidentes laborales:

Cartagena-Colombia, 2009-2012. Ciencias Psicológicas, 10(1), 31-41.

http://www.scielo.edu.uy/scielo.php?pid=S168842212016000100004\&script=sci abstract

Adeyemi, H. O., Adejuyigbe, S. B., Adetifa, B. O., Akinyemi, O. O. y Martins, O. O. (2020). Safe lifting ergonomics program for truck-loaders in Nigerian block making industries: A multi-site case study with qualitative and econometric analyses. Scientific African, 8, e00317. https://doi.org/10.1016/j.sciaf.2020.e00317

Álvarez Enciso, L. P., Mendoza Rincón, J., \& Navarro Oquendo, L. M. (2019). Costo/Beneficio como estrategia para la toma de decisiones del SG-SST para el sector hotelero de la localidad de Usaquén [tesis de especialización]. Corporación Universitaria Minuto de Dios. https://repository.uniminuto.edu/handle/10656/8160

Álvarez Torres, S. H., y Riaño Casallas, M. I. (2018). La política pública de seguridad y salud en el trabajo: el caso colombiano. Revista Gerencia y Políticas de Salud, 17(35), 111-131. https://doi.org/10.11144/javeriana.rgps17-35.ppss 
Arboleda Giraldo, M. (2019). Incidencia de los costos de prevención del Sistema de Gestión de Seguridad y Salud en el Trabajo (SG-SST) en el presupuesto general de una edificación en el Valle de Aburrá [tesis de maestría].

https://repositorio.unal.edu.co/handle/unal/59753

Bestraten Belloví, M., Gil Fisa, A., y Piqué Ardanuy, T. (2001). NTP 594: La gestión integral de los accidentes de trabajo (III): Costes de los accidentes. Ministerio de Trabajo y Asuntos Sociales. https://saludlaboralydiscapacidad.org/wpcontent/uploads/2019/0.5/NTP-594-La-gesti\%C3\%B3n-integral-de-los-accidentesde-trabajo-3-costes-de-los-accidentes.pdf

Bianchini, A., Donini, F., Pellegrini, M., y Saccani, C. (2017). An innovative methodology for measuring the effective implementation of an Occupational Health and Safety Management System in the European Union. Safety Science, 92, 26-33. https://doi.org/10.1016/j.ssci.2016.09.012

Bolaño Fandiño, J. (2019). Diseño de un método de estimación de costos generados por los accidentes de trabajo [tesis de maestría]. http://repository.unilibre.edu.co/handle/10901/17691

Carvajal Peláez, G. I., y Pellicer Armiñana, E. (2011). Propuesta para la evaluación del impacto económico de la siniestralidad laboral en el sector de la construcción. Revista Ingenierías Universidad de Medellín, 10(19), 89-99. http://www.scielo.org.co/scielo.php?script=sci_abstract\&pid=S1692$33242011000200009 \& \operatorname{lng}=\mathrm{es} \& \mathrm{nrm}=\mathrm{iso}$

Cuervo Vahos, A. (2019). Selección de un modelo de estimación del costo de un accidente de trabajo en Colombia a partir de la construcción del estado del arte de los modelos establecidos a nivel mundial [tesis de pregrado en Ingeniería Industrial]. Universidad Industrial de Santander.

Decreto 1072 de 2015 (26 de mayo), por medio del cual se expide el Decreto Único Reglamentario del Sector Trabajo. Diario oficial 49 523. http://www.suinjuriscol.gov.co/viewDocument.asp?ruta=Decretos $/ 30019522$

Díaz Rodríguez, A. V. (2019). Análisis de costos por accidentes de trabajo en las empresas del sector floricultor en el municipio de Tocancipá 2017-2018 [tesis de especialización]. http://repository.unimilitar.edu.co/handle/10654/31775 
Drummond, M. F., Sculpher, M. J., Claxton, K., Stoddart, G. L., y Torrance, G. W. (2015). Methods for the Economic Evaluation of Health Care Programmes. Oxford University Press. http://ebookcentral.proquest.com/lib/bibliotecaustaebooks/detail.action?docID $=4605509$

Franche, R.-L., Cullen, K., Clarke, J., Irvin, E., Sinclair, S., Frank, J., y The Institute for Work \& Health (IWH) Workplace-Based RTW Intervention Literature Review Research Team. (2005). Workplace-Based Return-to-Work Interventions: A Systematic Review of the Quantitative Literature. Journal of Occupational Rehabilitation, 15(4), 607-631. https://doi.org/10.1007/s10926-005-8038-8

Gil Fisa, A. (1999). NTP 540: Costes de los accidentes de trabajo: Procedimiento de evaluación. Ministerio de Trabajo y Asuntos Sociales. https://saludlaboralydiscapacidad.org/wp-content/uploads/2019/04/NTP540.pdf

Gil Fisa, A., y Pujol Senovilla, L. (2009). Metodología para la evaluación económica de los accidentes de trabajo. Prevención, trabajo y salud: Revista del Instituto Nacional de Seguridad e Higiene en el Trabajo, 7, 27-38. https://dialnet.unirioja.es/servlet/articulo? codigo $=1011386$

Grimaldi, J., y Simonds, R. (1996). La seguridad industrial: Su administración (2. ${ }^{a}$ ed.). Alfaomega.

Grimani, A., Bergström, G., Casallas, M. I. R., Aboagye, E., Jensen, I., y Lohela-Karlsson, M. (2018). Economic Evaluation of Occupational Safety and Health Interventions from the Employer Perspective: A Systematic Review. Journal of Occupational and Environmental Medicine, 6o(2), 147-166.

https://doi.org/10.1097/JOM.0000000000001224

Guirao Goris, J. A., Olmedo Salas, A., y Ferrer Ferrandis, E. (2008). El artículo de revisión. Revista Iberoamericana de Enfermería Comunitaria, 1(1), 1-25. https://www.uv.es/joguigo/valencia/Recerca files/el articulo de revision.pdf

Heinrich, H. W. (1931). Industrial accident prevention: A scientific approach. McGrawHill.

Jallon, R., Imbeau, D., y de Marcellis-Warin, N. (2011). Development of an indirect-cost calculation model suitable for workplace use. Journal of Safety Research, 42(3), 149-164. https://doi.org/10.1016/j.jsr.2011.05.006

Signos, Investigación en Sistemas de Gestión

ISSN: 2145-1389 | e-ISSN: 2463-1140 | DOI: https://doi.org/10.15332/24631140

Vol. 13 N.o 2 | julio-diciembre de 2021 
Junta de Castilla y León (Ed.). (2005). Aplicación de modelos de evaluación económica de las consecuencias de los accidentes del trabajo y enfermedades profesionales dirigidos a la medición de indicadores coste-eficacia y coste-beneficio en las actuaciones preventivas en la empresa, en el marco productivo de la comunidad autónoma de Castilla y León. Centro de Seguridad y Salud Laboral de Castilla y León. http://bibliotecadigital.jcyl.es/es/consulta/registro.cmd?id=20226

Lee, G. (2018). A Systematic Review of Occupational Health and Safety Business Cases. Workplace Health and Safety, 66(2), 95-104. https://doi.org/10.1177/2165079917730073

Leigh, J. P. (2011). Economic Burden of Occupational Injury and Illness in the United States. The Milbank Quarterly, 89(4), 728-772. https://doi.org/10.1111/j.14680009.2011.00648.x

Ley 1562 de 2012 (11 de julio), Por la cual se modifica el Sistema de Riesgos Laborales y se dictan otras disposiciones en materia de Salud Ocupacional. Diario oficial 48 4888. http://www.secretariasenado.gov.co/senado/basedoc/ley 1562 2012.html

Metis Gaia S. A. C. (2017). Diseño metodológico para el estudio de valoración económica en materia de seguridad y salud en el trabajo. Ministerio de Trabajo y Promoción de Empleo.

http://www.trabajo.gob.pe/CONSSAT/PDF/2018/Metodologia Valoracion Econ omica_pdf

Mossink, J. C. M. (2004). Comprender y aplicar el análisis económico en la empresa (Deborah Imel Nelson, Ed.). Organización Mundial de la Salud. https://www.who.int/occupational health/publications/ecoassessment/es/

Nadramija Nieva, N. D. (2018). Consultoría para la obtención del valor económico de los accidentes de trabajo en el Perú. https://cdn.www.gob.pe/uploads/document/file/302188/Estudio Valoraci\%C3\% B3n Econ\%C3\%B3mica 181116 MTPE VF 4 .pdf

Ramos, D., Arezes, P., y Afonso, P. (2016). Application of the Delphi Method for the inclusion of externalities in occupational safety and health analysis. DYNA, 83(196), 14-20. https://doi.org/10.15446/dyna.v83n196.56603.

Signos, Investigación en Sistemas de Gestión

ISSN: 2145-1389 | e-ISSN: 2463-1140 | DOI: https://doi.org/10.15332/24631140

Vol. 13 N.o 2 | julio-diciembre de 2021 
Resolución 0312 de 2019 (13 de febrero), por la cual se definen los Estándares Mínimos del Sistema de Gestión de la Seguridad y Salud en el Trabajo SG-SST. Diario oficial 50872.

http://legal.legis.com.co/document/Index?obra=legcol\&document=legcol 46de19 6963114b319f6f857c71866976

Riaño Casallas, M. I. (2017). Economía de la salud y seguridad en el trabajo: Un análisis de costos y beneficios desde las perspectivas del asegurador y de la empresa [tesis de doctorado]. Universidad Nacional de Colombia http://bdigital.unal.edu.co/60954/

Riaño Casallas, M., y Palencia Sánchez, F. (2015). Los costos de la enfermedad laboral: Revisión de literatura. Revista Facultad Nacional de Salud Pública, 33(2), 218227. https://doi.org/10.17533/udea.rfnsp.v33n2ao9

Riaño Casallas, M., y Palencia Sánchez, F. (2016). Dimensión económica de la seguridad y la salud en el trabajo: Una revisión de literatura. Revista Gerencia y Políticas de Salud, 15(30), 24-37. https://doi.org/10.11144/Javeriana.rgyps15-30.dess

Riaño Casallas, M., y Tompa, E. (2018). Cost-benefit analysis of investment in occupational health and safety in Colombian companies. American Journal of Industrial Medicine, 61(11), 893-900. https://doi.org/10.1002/ajim.22911

Rincón Ramírez, C. A., y Delgado Villarreal, P. M. (2018). Análisis de los costos de accidentalidad y enfermedad laboral en una compañía metalmecánica en Bogotá [tesis de especialización]. http://repository.udistrital.edu.co/handle/11349/14597

Steel, J., Godderis, L., y Luyten, J. (2018a). Methodological Challenges in the Economic Evaluation of Occupational Health and Safety Programmes. International Journal of Environmental Research and Public Health, 15(11). https://doi.org/10.3390/ijerph15112606

Steel, J., Godderis, L., y Luyten, J. (2018b). Productivity estimation in economic evaluations of occupational health and safety interventions: A systematic review. Scandinavian Journal of Work, Environment and Health, 44(5), 458-474. https://doi.org/10.5271/sjweh.3715 
Tompa, E., De Oliveira, C. y Dolinschi, R. (2006). Practice and potential of economic evaluation of workplace-based interventions for occupational health and safety. Journal of Occupational Rehabilitation, 16(3), 367-392. https://doi.org/10.1007/s10926-006-9035-2

Tompa, E., De Oliveira, C., Dolinschi, R., y Irvin, E. (2008). A systematic review of disability management interventions with economic evaluations. Journal of Occupational Rehabilitation, 18(1), 16-26. https://doi.org/10.1007/s10926-0079116-X

Tompa, E., Dolinschi, R., y Laing, A. (2009). An economic evaluation of a participatory ergonomics process in an auto parts manufacturer. Journal of Safety Research, 4O(1), 41-47. https://doi.org/10.1016/i.jsr.2008.12.003

Tompa, E., Dolinschi, R., y Natale, J. (2013). Economic evaluation of a participatory ergonomics intervention in a textile plant. Applied Ergonomics, 44(3), 480-487. https://doi.org/10.1016/j.apergo.2012.10.019

Tompa, E., Verbeek, J., Van Tulder, M., y de Boer, A. (2010). Developing guidelines for good practice in the economic evaluation of occupational safety and health interventions. Scandinavian Journal of Work, Environment \& Health, 36(4), 313318. https://doi.org/10.5271/sjweh.3009

Torres Navarro, C., y Callegari Malta, N. (2016). Criterios para cuantificar costos y beneficios en proyectos de mejora de calidad. Ingeniería Industrial, 37(2), 151-163. http://scielo.sld.cu/scielo.php?script=sci arttext\&pid=S181559362016000200005

Uegaki, K., de Bruijne, M. C., Lambeek, L., Anema, J. R., Van der Beek, A. J., Van Mechelen, W., y Van Tulder, M. W. (2010). Economic evaluations of occupational health interventions from a corporate perspective-A systematic review of methodological quality. Scandinavian Journal of Work, Environment \& Health, 36(4), 273-288. https://doi.org/10.5271/sjweh.3017

Universidad Pedagógica Nacional. (2017). Guía para la elaboración de resúmenes analíticos en educación-RAE. http://mpp.pedagogica.edu.co/download.php?file=odp 6589.pdf 
Van Dongen, J. M., Tompa, E., Clune, L., Sarnocinska-Hart, A., Bongers, P. M., Van Tulder, M. W., Van der Beek, A. J., y Van Wier, M. F. (2013). Bridging the gap between the economic evaluation literature and daily practice in occupational health: A qualitative study among decision-makers in the healthcare sector. Implementation Science: IS, 8, 57. https://doi.org/10.1186/1748-5908-8-57

Van Dongen, J. M., Van Wier, M. F., Tompa, E., Bongers, P. M., Van der Beek, A. J., Van Tulder, M. W., y Bosmans, J. E. (2014). Trial-Based Economic Evaluations in Occupational Health. Journal of Occupational and Environmental Medicine, 56(6), 563-572. https://doi.org/10.1097/JOM.0000000000000165

Vanegas Serna, D. (2014). Método para estimar el impacto económico de los accidentes de trabajo en los principales indicadores financieros [tesis de maestría]. Universidad EAFIT. http://repository.eafit.edu.co/handle/10784/5101

Vélez Ávila, M. A. (2018). Análisis costo beneficio de la implementación de un programa de prevención de accidentes laborales en talleres automotrices de la ciudad de Azogues. http://dspace.ups.edu.ec/handle/123456789/16143

Zárate, V. (2010). Evaluaciones económicas en salud: Conceptos básicos y clasificación. Revista médica de Chile, 138, 93-97. https://doi.org/10.4067/So03498872010001000 